\title{
Les mouvements de versant de Séchilienne (Isère) Prise en compte de l'héritage structural pour leur simulation numérique
}

P. POTHÉRAT P. ALFONSI

Laboratoire central des ponts et chaussées 58, bd Lefebvre, 75015 Paris Cedex potherat@lcpc.fr alfonsi@icpc.fr
Les instabilités du versant de Séchilienne (Isère, France) se rapportent à deux types de mécanismes : I'éboulement du couloir des $\alpha$ Ruines » et l'affaissement sommital du Mont Sec.

Les terrains mobilisés appartiennent à la $\propto$ série satinée ), ancienne formation gréso-pélitique métamorphisée dans le faciès schistes verts. L'étude structurale, nécessaire à la compréhension de la dynamique des mouvements de versant, a mis en évidence cinq phases majeures dont la plus importante dans la genèse des désordres actueis est la phase D4. tardihercynienne. Cet épisode tectonique, caractérisé par un système de décrochements conjugués de directions $\mathrm{N} 20^{\circ}$ à $\mathrm{N} 40^{\circ}$ et $\mathrm{N} 120^{\circ}$ à $\mathrm{N} 140^{\circ}$, est remarquable par la formation d'un grand pli d'axe NS lié à la mise en place d'un champ filonien à plomb-zinc. Les filons de quartz et les discontinuités associées respectent une disposition concentrique, en cônes inverses emboîtés ; ces structures jouent un rôle majeur dans l'évolution du massif. La phase D5, d'âge alpin, se caractérise par des plis d'entrainement engendrés par la réactivation des décrochements mis en place lors des phases précédentes. La simulation numérique des mouvements de versant, réalisée à l'aide de la méthode aux éléments distincts utilisée dans le code de calcul UDEC, s'est appuyée sur cette connaissance structurale du massif. Elle permet d'obtenir des résultats recoupant les données d'observation de la topographie actuelle et des déplacements verticaux.

Mots-clés : roche, versant, instabilités, éboulements, affaissement, glacier, analyse structurale, décrochements, antiforme, filons de quartz, cônes emboîtés, tardihercynien, alpin, simulation numérique, éléments distincts.

\section{The movements of the Séchilienne slope (Isère) Consideration of the structural setting for the numerical simulation}

The instabilities of the Sechilienne slope (Isère. French Alpes) are linked to two different kinds of mechnisms: the "Ruines $n$ rock falls and the subsidence of the upper part of the 4 Mont Sec $x$.

The rocks forming the moving slope belong to the "seirie satinée 3 , an old formation made of sandstones and pelitic layers. metamorphised into the " green schist $x$ facies.

The structural analysis helps to understand the mechanisms of the slope movements. It underlined five major stages, the most important one refers to the ground motion is the 4 th phase of late hercyrian age.
} 
This tectonic event, characterised by two conjugated shear zones respectively orientated $\mathrm{N} 020^{\circ}$ to $\mathrm{N}^{\circ} 40^{\circ}$ and $\mathrm{N} 120^{\circ}$ to $\mathrm{N} 140^{\circ}$, is remarkable by the raising of a large fold. That antiform, whose axis plunges to the North is linked to a leadzinc bearing quartz vein field.

The quartz veins and their associated fractures show a cone sheet structure and are subsequently of major importance in the slope evolution.

The 5 th tectonic stage, of alpine age, is characterised by folding along wrench fatilts due to the reactivation of the former fractures.

Numerical simulation was achieved by using distinct element modelling method (UDEC code) on the basis of the knowledge of the rock mass structure, The results obtained are in agreement with regard to the observed topography and vertical displacements.

Key words : rok siope, rock fall, subsidence, glacier, strike slip fautt, antiform, quartz veins, cone sheet, late hercynian, alpine, numerical simulation, distinct element method.

\section{1}

\section{Introduction}

Les mouvements de terrain observables sur le versant rive droite de la Romanche, entre Vizille et Séchilienne (Isère, France), se produisent depuis toujours sur le secteur des "Ruines», couloir d'éboulement actíf à l'origine de fréquentes chutes de blocs sur la RN 91 passant en pied de versant.

A partir de l'hiver 1985 l'accélération des phénomènes d'instabilité a conduit à mettre en place une surveillance en continu qui a permis de mettre en évidence l'extension des déformations à l'ensemble du versant (Antoine et al., 1987). Parmi les scénarii extrêmes envi- sageables, l'éboulement d'une masse de vingt millions de mètres cubes aurait des conséquences dramatiques pour la vallée de la Romanche (Évrard et al., 1990).

L'évaluation des risques d'éboulement en masse du versant, passe par la compréhension des mécanismes mis en jeu, notamment dans l'affaissement sommital et, en priorité, par une reconnaissance géologique affinée du site.

L'objectif principal d'une telle reconnaissance est de mettre en regard les événements tectoniques s'étant succédé sur le site et la genèse des désordres observés, partant de l'hypothèse que ces derniers constituent la réponse de l'héritage structural aux sollicitations exercées sur le versant par le biais du retrait du glacier de la Romanche.

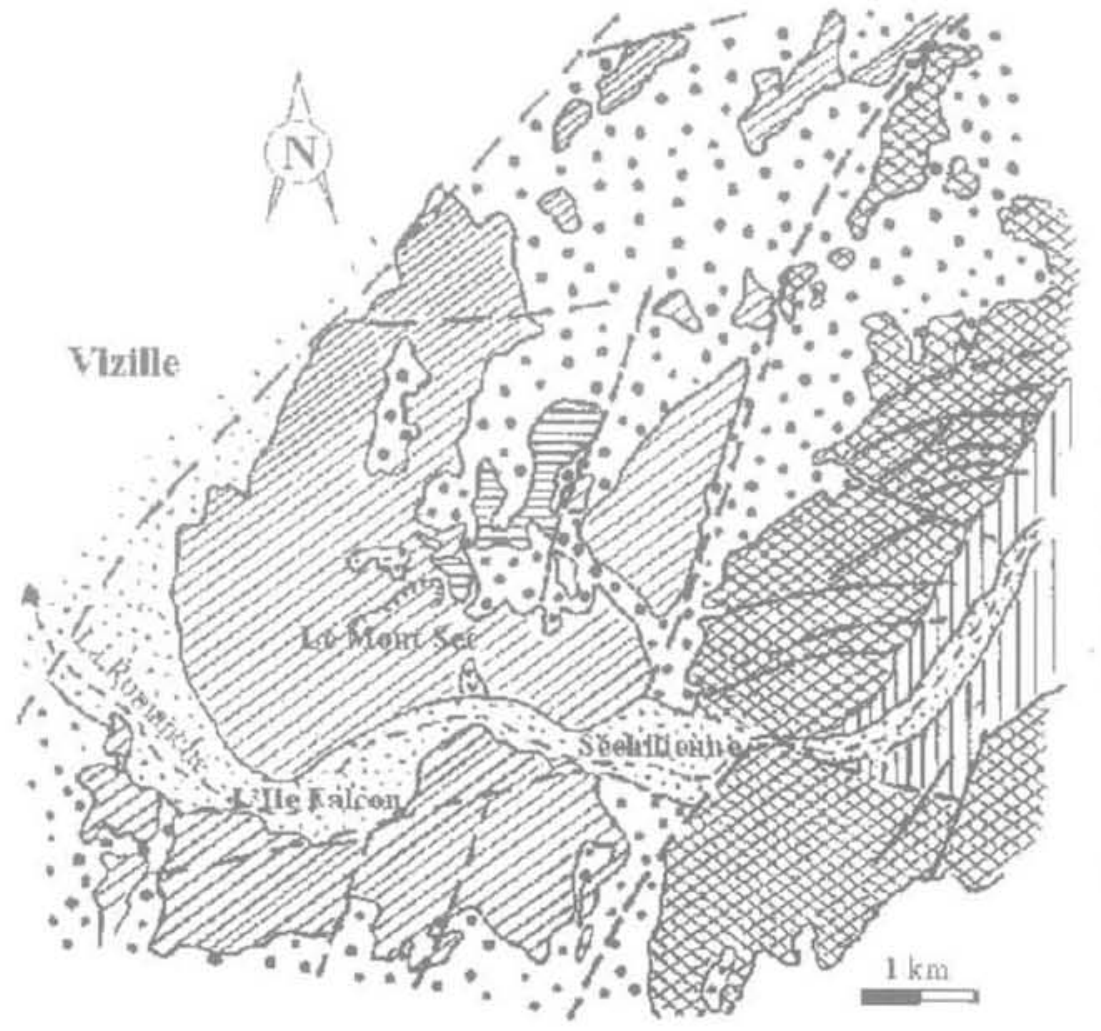

\section{Légende}

$\therefore$ Alluvions

$\because$ Glaciaire el cònes de déjectious

Couverure triassico-linsique

CO Micaschistes à denx micas du Rameau Ex temue de Belledoum

IIIIII Gneiss du complexe de Belledonne

Amphibolites feldspathiques du complexe de Belledome

fi. Eboulement des "kuines"

Faille priusipale

FIG. 1 Géologie de la région de Séchilienne (d'après la carte géologique au 1/50 000 de Vizille). The geological setting of the Séchilienne region (from geological map of Vizille at 1/50,000 scale). 


\section{Cadre géographique et géologique}

Situé $15 \mathrm{~km}$ au sud-est de Grenoble, dans les Alpes françaises, le versant en mouvement fait partie du "rameau externe $)$ de la chaine de Belledonne, caractérisé par la présence d'une bande de micaschistes (ou ("série satinée »), non précisément datée, encadrée à l'ouest par la couverture jurassique et la dépression d'Uriage et à l'est par une série cristallophyllienne basique en position inverse appartenant au « Rameau Interne ) de Belledonne (Fig. 1).

Deux accidents majeurs, de direction $\mathrm{N}^{\circ} 0^{\circ}$, soulignent les contacts entre ces différentes unités : l'accident de Vizille et le synclinal médian. Quelques lambeaux de terrains du Trias, du Lias et du Quaternaire reposent çà et là sur la " série satinée n.

\section{3}

\section{La succession lithologique}

\section{Les terrains de couverture}

\section{5}

\section{Le Quaternaire}

Représenté par les importants placages glaciaires du Würm, le Quaternaire est absent au niveau du glissement. En revanche il occupe les sommets (à l'est de Vaulnaveys) où il oblitère une partie des contacts et des structures. La géomorphologie du site ainsi que des stries d'origine glaciaire, observables sur quelques affleurements dans la zone active, attestent du recouvrement ancien du versant par le glacier de la Romanche.

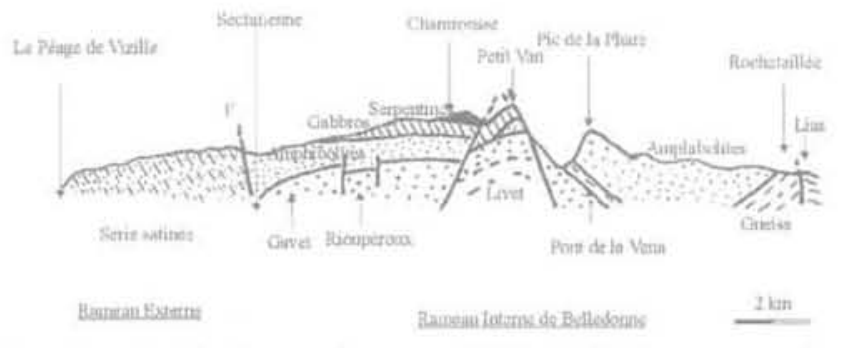

FG. 2 Coupe du massif de Belledonne par la vallée de la Romanche, entre Vizille et Rochetaillée (extrait du Guide géologique régional, Alpes du Dauphiné, 1983).

Cross-section of the Belledonne massif, through the Romanche valley, from Vizille to Rochetaillée (from Guide géologique régional, Alpes đu Dauphiné, 1983).

\section{1 .2}

\section{Les formations secondaires}

Des schistes pélitiques argileux noirs à bruns de l'Aalénien et des calcaires du Toarcien-Sinémurien surmontent une série réduite du Trias, composée de cargneules et gypses du Keuper reposant sur les cargneules et dolomies du Muschelkalk (au nord de Fonafrey).

\section{2}

\section{Les terrains paléozoïques}

\section{Le Carbonifère}

Directement au nord des « Ruines » de Séchilienne, des copeaux stéphano-westphaliens affleurent dans des fonds de synclinaux pincés d'axes NS à pendage vers le nord.

\section{9}

\section{Le Paléozoïque inférieur}

\section{- La « série satinée »}

Des micaschistes à deux micas, des chloritoschistes et séricitoschistes ou psammites, avec quelques passées concordantes de schistes graphiteux, admettent en leur sein quelques niveaux de prasinite et ovardite (roches métamorphiques de couleur vert foncé, respectivement à albite et à albite plus chlorite dominantes).

Ces terrains correspondent à une ancienne sẻrie gréso-pélitique en couches régulièrement alternées, d'âge probable Paléozoïque inférieur, métamorphisée dans les conditions du faciès des schistes verts.

\section{- Les séries cristallophylliennes basique et acide \\ Constituant un ensemble éruptif basique à serpen- tines et pyroxénites à la base, puis à gabbros surmontés d'une alternance de produits éruptifs basiques et acides et de niveaux sédimentaires, elles seraient à rapporter au Briovérien. Les deux séries affleurent en position inverse et sont ployées en une antiforme synclinale à grand rayon de courbure (Fig. 2).}

\section{L'héritage structural}

La reconstitution de l'histoire tectonique du versant de Séchilienne s'est appuyée sur l'examen des photos aériennes et sur des levés de terrain.

\section{1}

\section{La photo-interprétation}

L'interprétation des photos aériennes disponibles sur le secteur constitue la première étape de l'étude structurale, indispensable à la compréhension des 

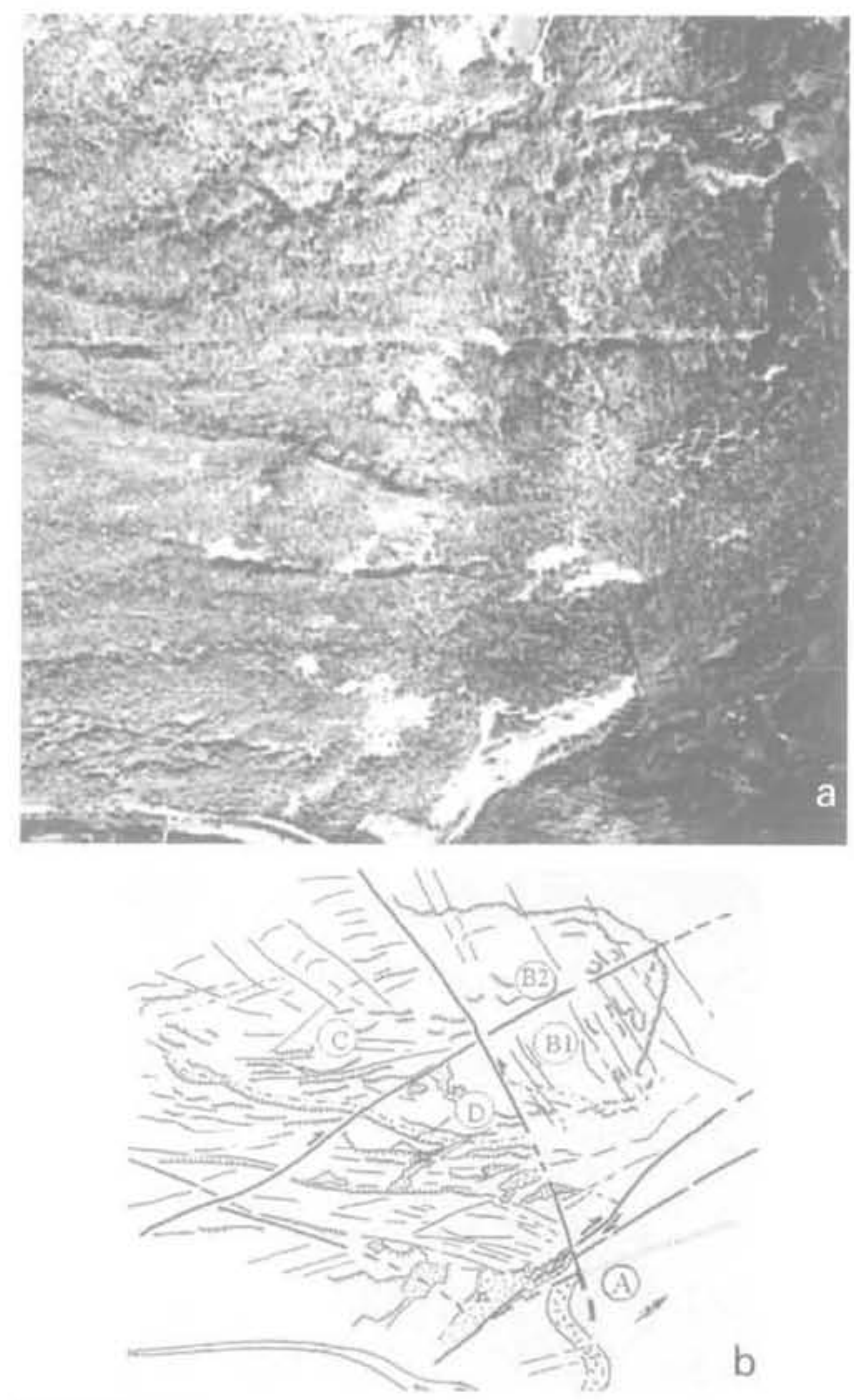

FIG. 3 Vue aẻrienne du versant de Séchilienne. a) L'affaissement sommital elliptique (photo Sintégra, 1986). b) Esquisse structurale (les lettres désignent des panneaux à cinématique variable).

Aerial view of the Sèchilienne slope. a) Elliptical upper settling zone. b) Structural sketch (the surrounded letters show different moving areas)

mécanismes mis en jeu dans le mouvement de versant. Elle permet d'abord de repérer les méga structures (grands plis, accidents majeurs, zones broyées) puis d'établir une esquisse de schéma structural.

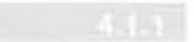 \\ La fracturation}

Du schéma structural tiré de l'étude des photos aériennes (couverture à 1/10 000, réalisée en 1986), se dégagent quatre familles de discontinuités (Fig. 3a et 3b) :

- failles de direction $\mathrm{N} 20^{\circ} \mathrm{a} \mathrm{N} 30^{\circ}$, plus ou moins parallèles à la direction du synclinal médian qui représente le chevauchement du rameau interne de Belledonne sur le rameau externe (série satinée). Leur jeu est illustré par la virgation, à valeur de décrochement dextre, sement du mont Sec (Grande Combe) au passage de la faille principale $\mathrm{N} 20^{\circ}$ qui traverse l'affaissement ;

- failles $\mathrm{N} 120^{\circ}$ à $\mathrm{N} 140^{\circ}$ conjuguées des précédentes, jouant en décrochements senestres comme le montre le décalage vers le NW, par le jeu de l'accident $\mathrm{N} 140^{\circ}$ du sommet des "Ruines », de la partie NE de l'affaissement sommital. Ce mouvement se manifeste par la brutale interruption du sillon de la Grande Combe qui paraît décalé vers la gauche d'environ $80 \mathrm{~m}$, au même titre que les falaises bordières au sud de l'affaissement;

- au niveau du couloir des « Ruines n un important accident N-S semble avoir rejoué en queue de décrochement dextre au vu de la disposition des failles qui lui sont associées et du crochon apparent dessiné par un niveau repère visible en bordure est du couloir d'éboulis :

- enfin, une direction $\mathrm{N}^{\circ} 0^{\circ}$ qui n'apparait que dans le versant du mont Sec, est associée à la présence des filons de quartz des « Ruines » et de la Grande Combe.

Parmi ces familles de discontinuités, trois accidents importants (en gras sur la figure $3 \mathrm{~b}$ ) découpent le massif en compartiments différemment structurés :

- compartiment A : à l'est du couloir des « Ruines » la fracturation est moins dense et on n'y retrouve pas les fractures $\mathrm{N} 70^{\circ}$ à $\mathrm{N} 80^{\circ}$;

- compartiment B (B1 + B2) : au NE du décrochement $\mathrm{N} 140^{\circ}$, ce compartiment est découpé en lanières par des accidents de même nature et même direction ; - compartiment $\mathrm{C}$ : les discontinuités $\mathrm{N} 20^{\circ}$ et $\mathrm{N} 70^{\circ} \mathrm{y}$ sont mieux représentées que les discontinuités $\mathrm{N} 140^{\circ}$, presque absentes ;

- compartiment D : dans le dièdre sud, délimité par les failles $\mathrm{N} 20^{\circ}$ et $\mathrm{N} 140^{\circ}$, les accidents $\mathrm{N} 20^{\circ}$ et $\mathrm{N} 70^{\circ}$ à $\mathrm{N} 90^{\circ}$ sont nombreux alors que les accidents N140 n'apparaissent plus.

Ce découpage du massif est compatible avec la délimitation de panneaux à cinématique différente, effectuée d'après l'analyse des trajectoires des balises de surveillance.

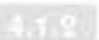

\section{L'affaissement du Mont Sec}

De forme grossièrement elliptique, il est bordé de discontinuités concentriques et recoupé par quelques fractures rayonnantes qui convergent vers le centre de l'ellipse. Ce dispositif, en étroite relation spatiale avec un pli à grand rayon de courbure et avec la mise en place du champ filonien de Séchilienne, évoque les structures habituellement observées dans l'encaissant des formations affectées par un magmatisme anorogénique, où les failles radiales ainsi que les discontinuités concentriques à pendage centripète (cone sheet) représentent des fractures de cisaillement générées par le bombement du massif lors de l'expansion du magma (Fig. 4).

\section{2}

\section{Les levés de terrain}

Les différentes générations de plis, les failles et les microstructures (schistosités, diaclases, joints, stries, linéations...) furent identifiées, analysées et replacées dans leur ordre chronologique en accord avec l'état actuel des connaissances de la géologie alpine (Guillot, 2000), 


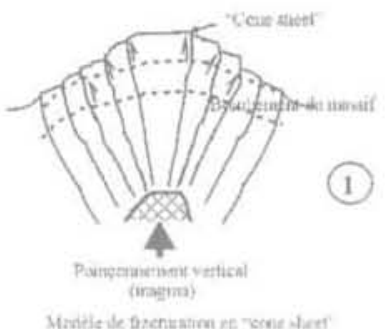

(1)

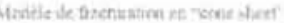

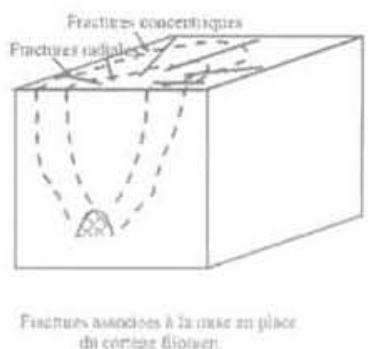

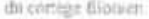

FiG.4 Modèle de genèse de fractures radiales et concentriques par poussée verticale, associée à la mise en place d'un réseau de filons de quartz.

Genesis modelling of radial and concentric fractures caused by a vertical upward thrust and linked to a quartz vein system.

\section{1}

\section{Les phases de déformation précoces $\left(D_{1}, D_{2}\right)$}

La première phase de déformation (D,), responsable de la foliation est accompagnée de la genèse de filonnets de quartz d'exsudation synschisteux. On ne connaît pas de pli associé à cet épisode.

La série est ensuite plissée lors d'une phase de déformation $\left(D_{2}\right)$, caractérisée par l'apparition des plis les plus anciens repérés $\left(\mathrm{P}_{2}\right)$ qui reprennent les filonnets de quartz synschisteux. Il s'agit de petits plis isoclinaux à axe vertical, observés en plusieurs points du versant. Quelques plis en genou, à axe également vertical et d'amplitude décamétrique sont à rapporter à cette même phase de déformation (Fig. 5). La schistosité $\mathrm{S}_{1}$ est confondue avec $\mathrm{S}_{0}$.

La phase de déformation $D_{1}$ serait antécarbonifère tandis que la phase $D_{2}$ correspondrait au début de l'orogénie hercynienne (antéwestphalienne).

\subsection{9}

\section{Les phases de déformation hercyniennes}

\section{- La phase $D_{3}$}

Une phase hercynienne (westphalienne?), à l'ori-

gine de l'empilement des couches des unités du complexe de Belledonne, provoque le chevauchement à vergence sud de l'ensemble basique de Chamrousse sur la formation du Taillefer et se traduit par la genèse de plis $\mathrm{P}_{3}$,

Les plis $\mathrm{P}_{3}$ sont des plis dissymétriques à flanc court subhorizontal et à flanc long subvertical (Fig. 6), légèrement penté vers le nord (flanc inverse d'un grand pli à vergence sud ?). Les axes horizontaux ont une direction $\mathrm{N} 50^{\circ}$ à $\mathrm{N} 80^{\circ}$.

Certains accidents $N 60^{\circ}$ à $N 70^{\circ}$ à jeu sénestre sont contemporains de cette phase de déformation.

D'autres accidents conjugués $\mathrm{N} 20^{\circ}$ à $\mathrm{N} 50^{\circ}$ et $\mathrm{N} 120^{\circ}$ à $\mathrm{N} 140^{\circ}$ jouent en décrochements respectivement sénestres et dextres, ainsi qu'en attestent les tectoglyphes ornant les miroirs de failles.

\section{- La phase $\mathrm{D}_{4}$}

Une phase de déformation tardihercyniennne $\left(\mathrm{D}_{4}\right)$, a joué un rôle prépondérant dans la structuration du massif et continue vraisemblablement de jouer un rôle majeur dans la genèse des désordres observés à l'heure actuelle.

Cet épisode tectonique, initié en régime transcurrent (plis et décrochements associés), est caractèrisé par la genèse d'un grand pli d'axe N-S vraisemblablement associé à la mise en place d'un champ filonien à plomb-zinc (Fig. 7).

\section{- Les plis et discontinuités associées}

Au niveau de la galerie de mine (cote 900), une voussure, bien visible sur les photos du versant prises d'hélicoptère, constitue le trait structural majeur. La direction $\left(\mathrm{N} 20^{\circ}\right)$ et le plongement axial $\left(45^{\circ} \mathrm{N}\right)$ de cette antiforme ont été déterminés par projection stéréographique des plans de foliation relevés dans la recoupe ouest de la galerie d'exploitation (Fig. 8). D'après les attitudes de ces plans de foliation reportées sur le canevas structural, il semble que toute la partie de l'affaissement sommital, soit affectée par ce vaste pli (Fig. 9).

Des plis $\mathrm{P}_{4}$, d'ordre inférieur, d'axe horizontal N170 à $\mathrm{N}$-S, reprennent les plis $\mathrm{P}_{3}$. Certains d'entre eux sont associés à des failles inverses sur des accidents pentés vers l'ouest.

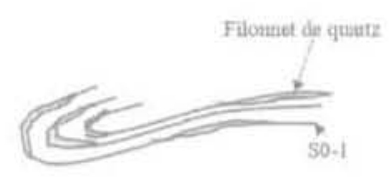

Quartz d'exsurlation synsclisteux, plissed Folded exuded quartz vein formed daring the foliation stage

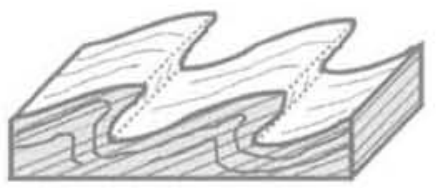

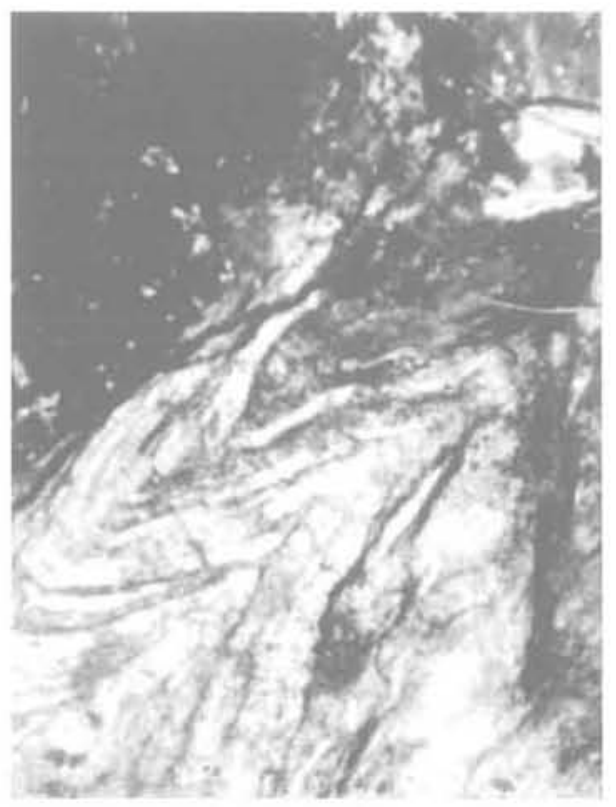

fic.5 Plis P2, verticalisés.

Verticalised P2 folds. 

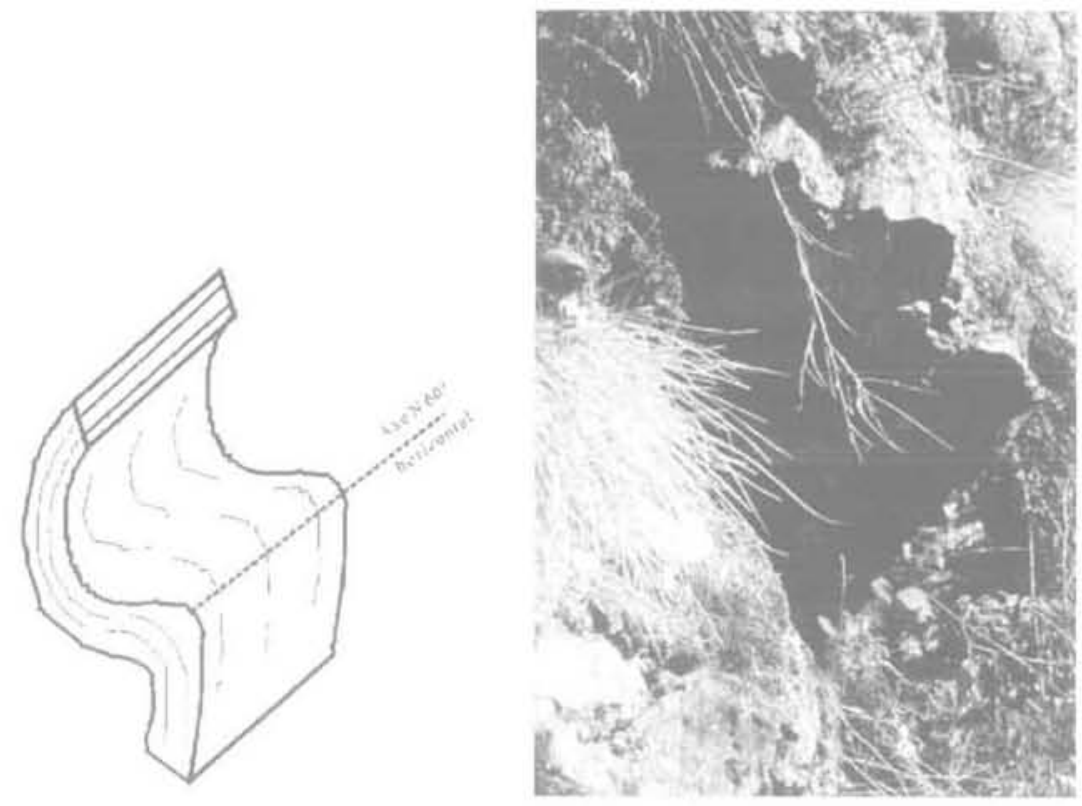

Les accidents conjugués $\mathrm{N} 20^{\circ}$ à $\mathrm{N} 50^{\circ}$ et $\mathrm{N} 120^{\circ}$ à $\mathrm{N} 140^{\circ}$, à nouveau sollicités, jouent en sens inverse du jeu précédent, donc en décrochements respectivement dextres et senestres. Deux d'entre eux ont une influence prépondérante dans le découpage du massif :

- le décrochement dextre $\mathrm{N}^{2} \mathrm{O}^{\circ}$ allant des Rivoirands à la bordure NNE de l'affaissement sommital ;

- le décrochement sénestre N140 formant la limite supérieure du couloir des « Ruines ».

\section{- Le champ filonien de Séchilienne}

Une des singularités de la série satinée, entre Vizille et Séchilienne, a trait à la présence de nombreux filons de quartz à plomb-zinc exploítés de 1850 à 1914. La nature et la similitude des paragenèses (associations minérales), la zonalité de la minéralisation, de mème que l'occurrence de dykes de lamprophyres dans la mine de Montjean qui suggèrent la présence en profondeur d'un corps granitoïde, plaident en faveur d'un remplissage synchrone de structures annulaires et radiales, par des solutions hydrothermales, au cours d'une période de bombement liée à un phénomène de plutonisme au droit du Mont Sec.

La distribution spatiale des filons, d'attitudes très variables, ne peut s'intégrer dans un schéma simple, en compression ou en distension (Fig. 10). Ces derniers s'organisent sur des cercles centrés sur l'affaissement sommital, avec généralement un pendage centripète, mis à part le filon de Montjean $\left(\mathrm{n}^{\circ} 4\right)$, orienté $\mathrm{N} 40^{\circ}$, soit selon une faille radiale.

Les relations spatiales entre la voussure du mont Sec et le champ filonien de Séchilienne incitent à penser que ces deux types de phénomènes résultent du même processus génétique, la mise en place de la minéralisation dans des fractures d'extension relevant de la remobilisation et du transfert chimique, par des fluides géothermaux, des métaux de l'encaissant ou des granitoídes précoces, sous l'action d'un flux de chaleur généré par la montée d'un cryptodôme magmatique.

Bien que l'épaisseur des filons exploités ne soit que de 2 à $3 \mathrm{~m}$, la largeur des caisses filoniennes peut atteindre $6 \mathrm{~m}$ et prouve que l'ouverture du massif a été considérable dans le versant de Séchilienne. Quel que soit l'âge des filons (probablement synchrone de l'émis- sion de trachy-andésites des Rousses, rapportées au Permien), la tectonique distensive, cogénère de leur mise en place, a donc largement contribué à affaiblir le massif en surimposant ses traits structuraux spécifiques à ceux préexistants et doit être prise en considération dans l'étude de stabilité du versant.

Les fractures associées à ce système respectent une disposition concentrique, en cônes inverses emboîtés ou " cone sheet ») (Adam, Gagny, 1989), confirmée, d'une part, par les observations de terrain (falaise arrière de l'affaissement du Mont Sec minéralisée. dépression de la Grande Combe et failles $\mathrm{N} 70^{\circ}$ bordées d'escarpements à regard amont), d'autre part, par les observations effectuées dans la galerie de reconnaissance (cote $710 \mathrm{~m}$ ) où de nombreuses fractures à pendage amont (vers le nord) ont été levées. Leur caractère précoce, ante-alpin, est souligné par la virgation à valeur de décrochement dextre subie par le sillon de la Grande Combe au passage de la faille principale $\mathrm{N} 20^{\circ}$ qui traverse l'affaissement (Fig. 3b) et par son interruption au contact du décrochement sénestre $\mathrm{N} 140^{\circ}$ du sommet des « Ruines » qui décale la structure elliptique du Mont Sec d'une centaine de mètres vers la gauche.

Ces discontinuités revêtent une importance majeure dans l'évolution du massif car les basculements observés à leur niveau dans la galerie de reconnaissance témoignent de leur action prépondérante dans la dynamique du mouvement du versant qui se manifeste par l'affaissement en coin du Mont Sec lié à la décompression du massif, consécutive au retrait du glacier de la Romanche.

\section{3}

\section{Les phases tardives}

\subsection{1}

\section{La phase de déformation alpine $\left(D_{5}\right)$}

Les plis $P_{5}$, sont représentés à toutes les échelles dans le versant de Séchilienne. Toujours associés aux décrochements $\mathrm{N} 20^{\circ}$ à $\mathrm{N} 50^{\circ}$ ou $\mathrm{N} 120^{\circ}$ à $\mathrm{N} 140^{\circ}$, ils possèdent un axe $\mathrm{N}^{\circ}$ à $\mathrm{N} 20^{\circ}$, fortement penté vers le nord, et peuvent être assimilés à des plis d'entrainement. 

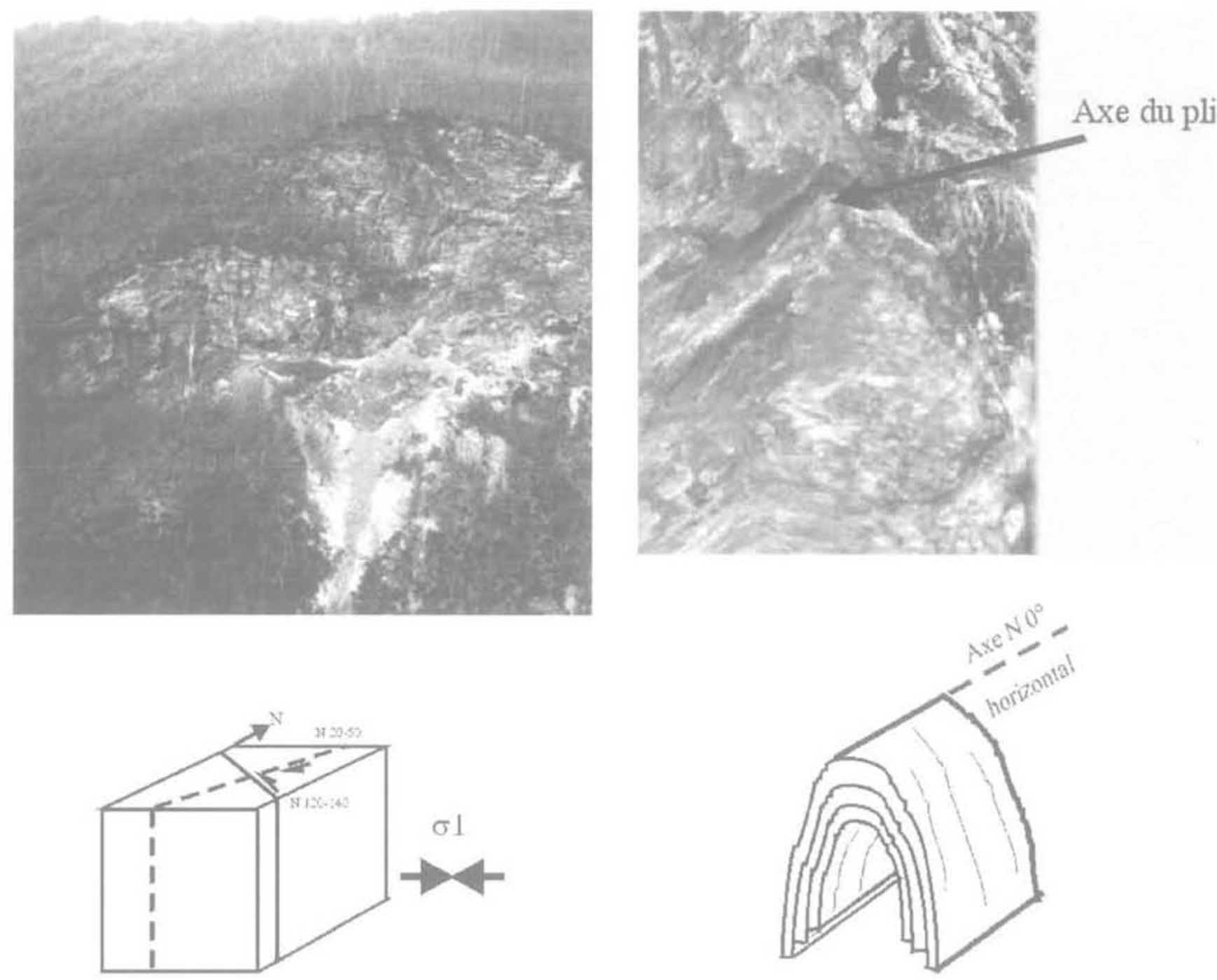

AG,7 Différents types de plis P4. a) Grand pli sommital P4 d'axe N20 , penté $45^{\circ}$ nord. b) Pli P4 de deuxième ordire, c) Système de décrochements conjugués. d) Petit pli P4 isoclinal.

Different P4 folds, a) Upper large $\mathrm{P} 4$ fold with a $\mathrm{N}^{\circ} \mathrm{O}^{\circ}$ axis plonging $45^{\circ}$ to the north. b) Minor $\mathrm{P} 4$ fold. c) Conjugated strike slip fault system. d) Minor isoclinal P4 fold.

engendrés par ces mouvements transcurrents (Fig. 11).

Les discontinuités les plus marquées sont les décrochements conjugués $\mathrm{N} 20^{\circ}$ et $\mathrm{N} 140^{\circ}$, respectivement dextres et sénestres, dont le jeu est attesté par les stries subhorizontales qui ornent les miroirs de failles. Les décrochements dextres sont prépondérants sur le flanc ouest du pli sommital, en particulier dans la galerie de reconnaissance où ils jouent dans la foliation. Les décrochements sénestres, en revanche, découpent en lanières le flanc est du pli sommital limité au SW par la fracture sommitale du couloir des « Ruines $»$, de direction $\mathrm{N} 140^{\circ}$.

Une autre famille de discontinuités est représentée par les plans de chevauchement fort bien exprimés dans la galerie de reconnaissance hydraulique située dans le versant opposé, rive gauche de la Romanche. De direction moyenne $\mathrm{N}$-S et de pendage $15^{\circ} \mathrm{E}$ à $20^{\circ} \mathrm{E}$, ces plans sont associés à des structures typiquement alpines : les anastomoses. Relayées par des plans faiblement pentés vers l'est, ces structures en fleur sont interprétées comme l'expression précoce du raccourcissement alpin par Effendiantz et Guillot (2000) et traduisent l'expulsion vers le haut du matériau comprimé en régime trans- pressif (Fig. 12). Le couloir des «Ruines», de direction N-S semble s'ètre formé sur l'une d'elles.

Le couloir des « Ruines ». La direction structurale N$\mathrm{S}$ est uniquement présente dans le couloir des "Ruines ), orienté selon celle-ci. L'abondance de discontinuités de ce type et la présence de môles résistants de forme losangique, découpés par des discontinuités $\mathrm{N}^{\circ}$ et $\mathrm{N} 120^{\circ}$, évoque une zone de cisaillement (shear zone) méridienne, de $80 \mathrm{~m}$ de large, au sein de laquelle se sont manifestés les premiers désordres. Le jeu senestre sur la faille $\mathrm{N} 140^{\circ}$ des « Ruines ») est tardif par rapport au fonctionnement de la shear zone N-S, décalée d'une centaine de mètres vers la gauche (Fig. 3b).

\section{$4+38$}

\section{Les indices de mouvements récents}

L'examen des documents cartographiques ou des photographies aériennes du secteur de Séchilienne, révèle au niveau de la vallée de la Romanche, à l'emplacement du village de Séchilienne, l'existence d'un petit bassin de forme losangique qui évoque l'allure d'un 


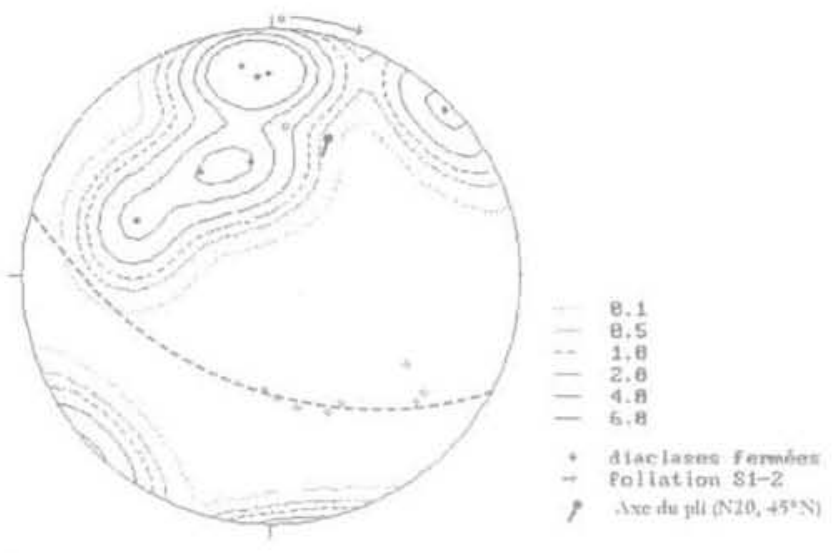

FG. 8 Le grand pli sommital du Mont Sec. The upper a Mont Sec w large fold.

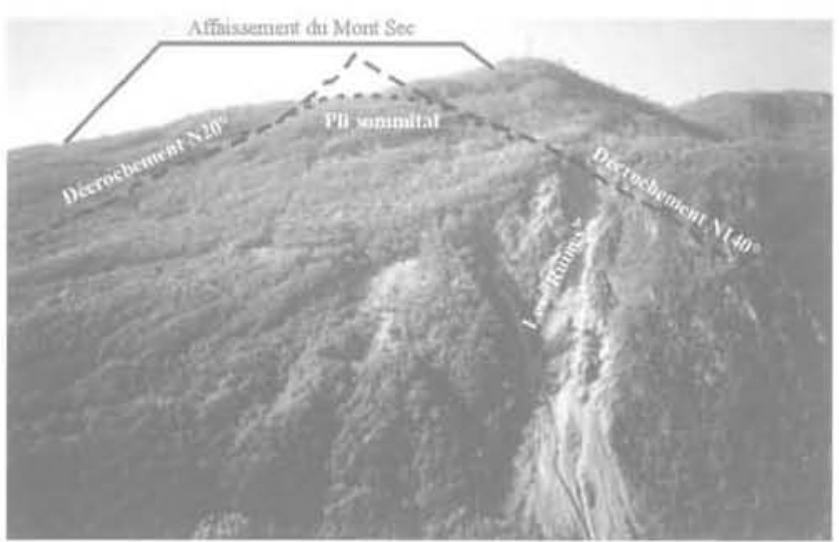

FIG.9 Vue du couloir des "Ruines » et de l'affaissement du Mont Sec.

View of the "Ruines n corridor and $\alpha$ Mont Sec subsidence. bassin de type pull-apart (Fig. 13). Les structures de type pull-apart sont caractéristiques des zones de cisaillement dans lesquelles certains tronçons de failles en relais entre deux failles décrochantes jouent en ouverture.

La réalité cartographique du jeu relatif des accidents $\mathrm{N} 110^{\circ}$ bordant le losange est indubitable à l'examen de la carte géologique à 1/50 000 de Vizille (Fig. 1) car les amphibolites sont décalées de manière sénestre d'environ $1 \mathrm{~km}$ par le segment nord d'une faille $\mathrm{N} 110^{\circ}$ passant dans le lit de la Romanche à la sortie est de Séchilienne. Les schistes de la "Série Satinée », dont certains pointements apparaissent dans le cône alluvial du torrent de Saint-Barthélemy de Séchilienne, sont décalés d'autant, toujours vers la gauche, par le segment sud. Le $\alpha$ synclinal médian $\cdots$, contact anormal entre les rameaux interne (amphibolites) et externe (" série satinée ») de Belledonne est donc lui aussi pris dans ce mouvement décrochant.

Le segment nord est parfaitement identifiable en bordure de la route de Bourg-d'Oisans, à la sortie de Séchilienne, par la présence de miroirs striés avec tectoglyphes indiquant un jeu dextre puis sénestre. En revanche le segment sud, masqué par les alluvions de la Romanche, est plus difficile à mettre en évidence. Cependant, en pied de versant, sur la gauche du couloir des «Ruines », une zone extrêmement broyée, caractérisée par la présence de miroirs striés ${\mathrm{N} 110^{\circ}}^{\circ}$ avec indices de mouvements sénestres, matérialise son passage tandis que le long de l'ancienne route, au pied du couloir d'éboulis, plusieurs répliques de cet accident découpent la falaise basale.

Bien que ces mouvements ne soient pas datés avec précision, plusieurs arguments plaident en faveur d'un rejeu récent de la faille de la Romanche :

- le remplissage par des matériaux quaternaires du bassin losangique, dont nous ne connaissons pas l'épaisseur réelle;

- la présence d'un talus rectiligne, parallèle à la direction de la faille de la Romanche, de $800 \mathrm{~m}$ de long pour

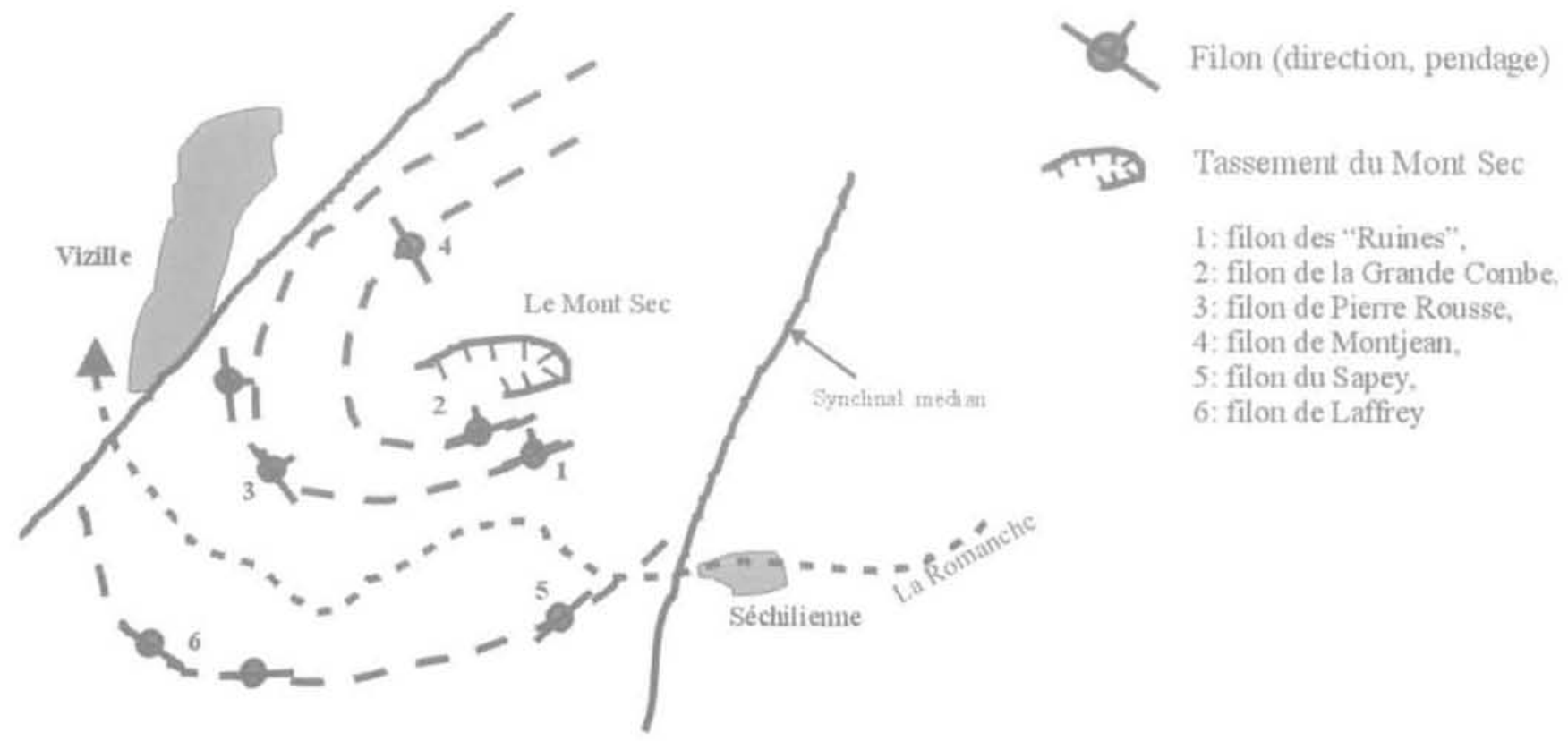

FG. 10 Relations spatiales entre le cortège filonien de Séchilienne et l'affaissement sommital du Mont Sec. Spatial relations between the Sèchilienne quartz vein system and the " Mont Sec n upper settling zone. 

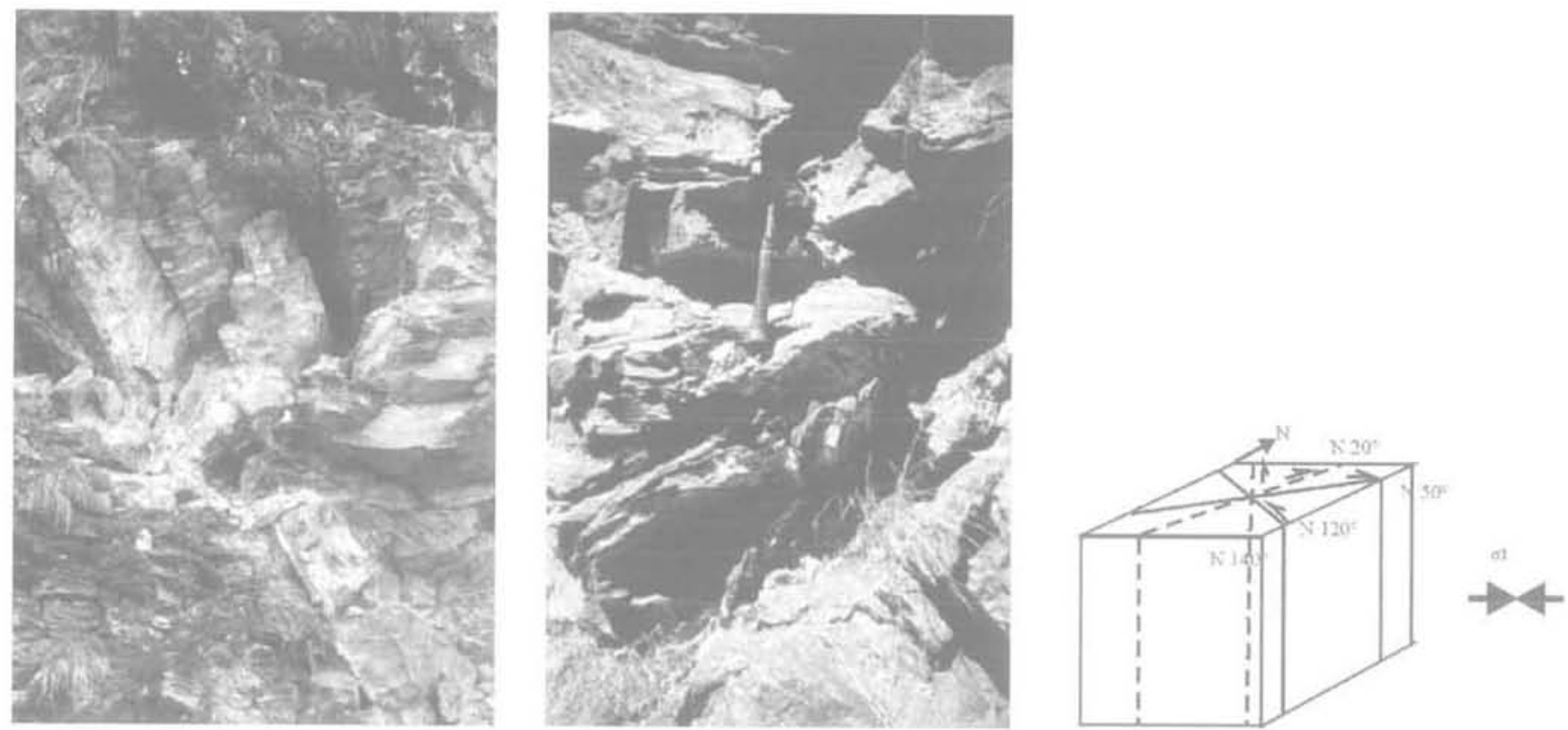

HG. 11 Les plis P5 et les décrochements associés. a) Pli d'entrainement sur décrochement senestre N140 d'entraînement sur décrochement dextre N20 . c) Systèmes de décrochements conjugués.

P5 folds and associated strike slip faults. a) Fold along a N140 sinistral strike slip fault. b) Fold along a N20 dextral strike slip fault. c) Conjugated strike slip faults system.

une vingtaine de mètres de hauteur, affectant le cône de déjection du torrent de Saint-Barthélemy de Séchilienne,

Reprise de l'activité du site dans les années 60-70, exprimée par des éboulements au niveau de la faille de la Romanche, en pied de versant et sur la faille sommitale du couloir des « Ruines ».

Le dernier argument, d'ordre sismique, est relatif au tremblement de terre de Laffrey du 11 janvier 1999. La réinterprétation des mécanismes au foyer (Thouvenot et al., en préparation) de cette secousse d'une magnitude 3,5 , a permis d'avancer l'hypothèse d'un jeu possible sur une faille E-W (qui pourrait être la faille de la Romanche ?). Se pose alors la question d'un éventuel rôle de la néotectonique dans la genèse des désordres observés (Pothérat et al., en préparation). Cette hypothèse demande à être confirmée (ou infirmée) par des investigations complémentaires à mener en particulier au niveau du remplissage alluvionnaire du petit bassin de Séchilienne.

\section{5}

\section{Impact de l'histoire tectonique sur l'évolution du versant de Séchilienne}

Les phases tectoniques $\mathrm{D}$, à $\mathrm{D}$, responsables de la structuration du Mont Sec, ont eu pour effet de générer des accidents conjugués de directions $\mathrm{N} 20^{\circ}$ à $\mathrm{N} 50^{\circ}$ et $\mathrm{N} 120^{\circ}$ à $\mathrm{N} 140^{\circ}$, de même que des couloirs de cisaillement privilégiés (shear zones) de direction $\mathrm{N}-\mathrm{S}$ tels que celui des $\propto$ Ruines $n$, et de les faire jouer en décrochements dextres ou sénestres en fonction de l'orientation de la contrainte principale. Lors de l'orogénie alpine
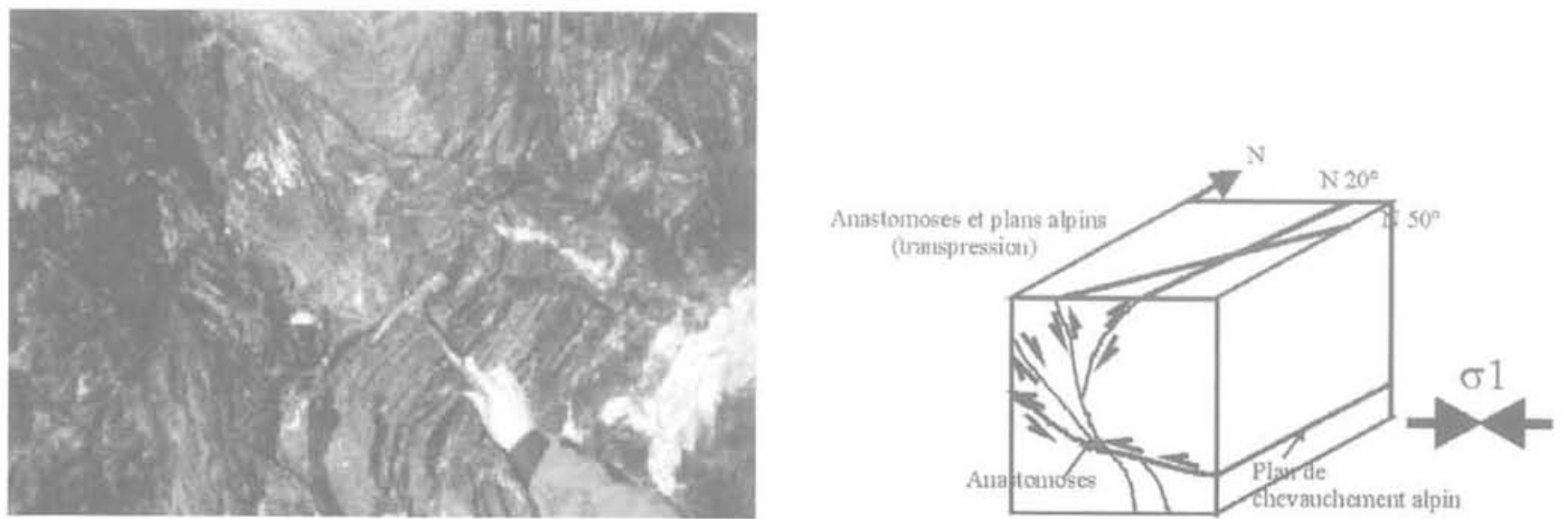

FG. 12 Relations entre les plans de chevauchements alpins et les anastomoses. a) Anastomoses dans la galerie de reconnaissance. b) Interprétation structurale.

Relation between alpine thrust planes and the " anastomosis v. a) Anastomosis in the exploration tunnel. b) Structural interpretation. 

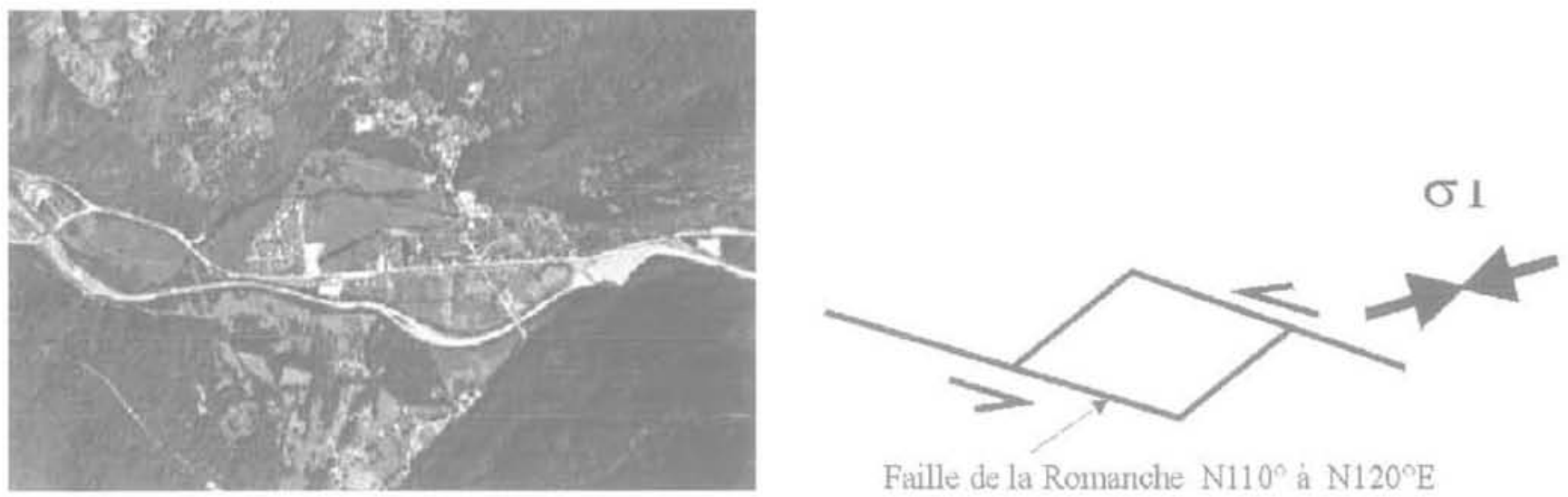

Faille de la Romanche $\mathrm{N} 110^{\circ}$ à $\mathrm{N} 120^{\circ} \mathrm{E}$

FG. 13 Le bassin losangique de Séchilienne. a) Vue aérienne du village de Séchilienne. b) Interprétation structurale.

The Séchilienne losangic basin. a) Aerial view of the Séchillenne village. b) Structural interpretation.

ces mouvements ont été facilités et amplifiés par une structure héritée de la phase tardihercynienne: le pli sommital du Mont Sec, d'axe $\mathrm{N}^{\circ} 0^{\circ}$.

Contemporaine de cette phase $\mathrm{D}_{4}$, la mise en place du cortège filonien de Séchilienne a surimposé ses traits spécifiques aux structures hercyniennes : fractures en cones sheet, de direction $\mathrm{N}^{\circ} 0^{\circ}$ à $\mathrm{N} 70^{\circ}$ sur sa bordure sud, fractures à disposition radiale et filons. Le massif est découpé en cinq compartiments $\left(\mathrm{A}, \mathrm{B}_{1}, \mathrm{~B}_{2}, \mathrm{C}\right.$ et D), différemment structurés par trois accidents principaux appartenant aux familles citées plus haut. La décompression récente du massif, responsable de l'affaissement en coin du Mont Sec, a contribué à révéler ces accidents soulignés, sur sa bordure sud, par un escarpement à regard amont de direction $\mathrm{N} 60^{\circ}$ à $\mathrm{N} 70^{\circ}$ et par le décalage sénestre de l'affaissement lui-même suivant le décrochement $\mathrm{N} 140^{\circ}$ du sommet des «Ruines ».

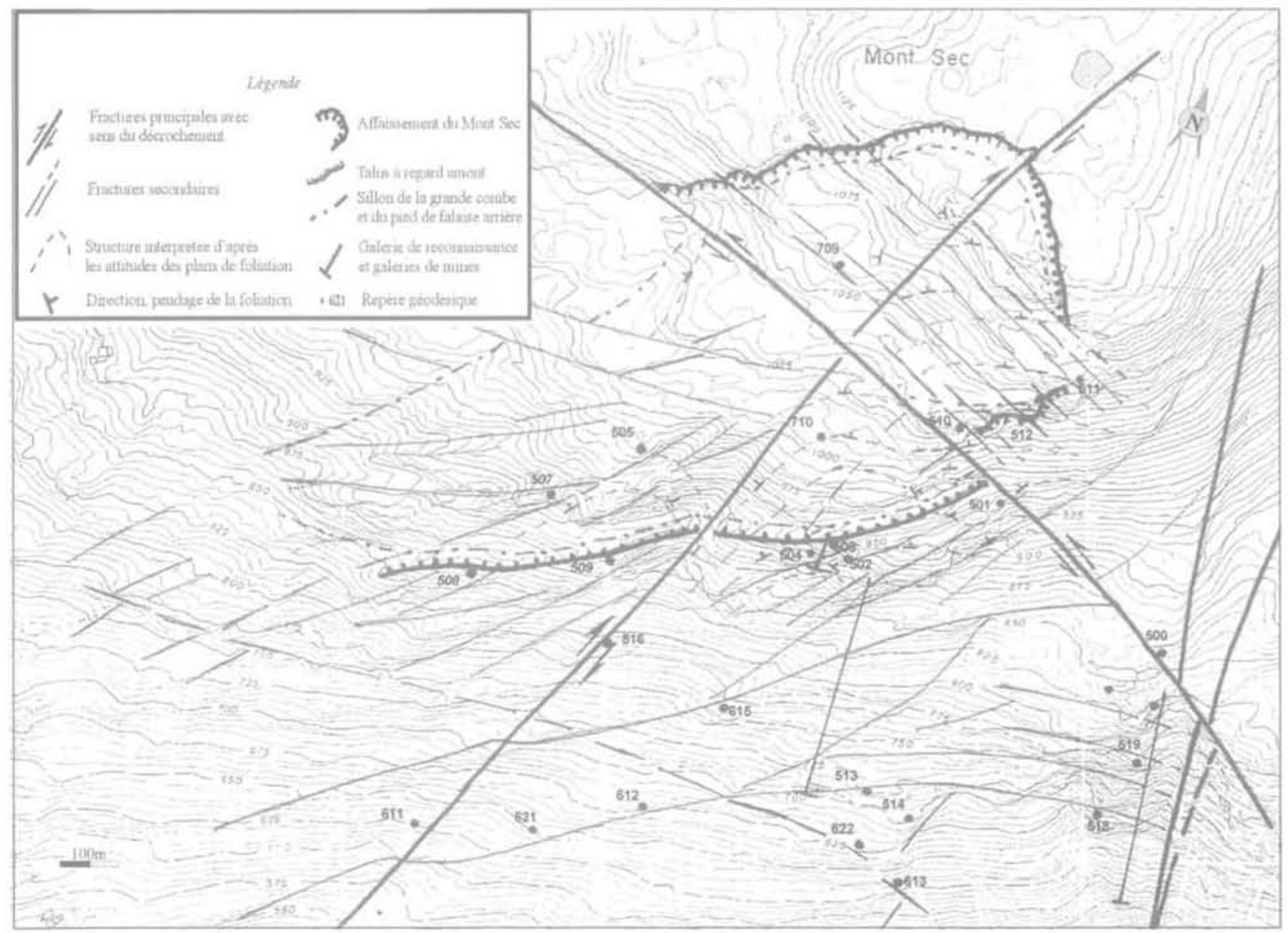

FIG. 14 Esquisse structurale du versant de Séchilienne. Structural sketch of the Séchilienne slope area. 
L'esquisse structurale, à 1/5 000 du secteur de Séchilienne, représentée sur la figure 14, illustre la différence de structuration entre les compartiments $\mathrm{B}_{1}$ et $\mathrm{B}_{2}$ d'une part, et C et D, d'autre part, à mettre en relation avec la position de la charnière du pli sommital. En effet, compte tenu de la présence de niveaux de schistes et pélites noires dans la série, les décrochements joueront préférentiellement dans la foliation $\mathrm{S}_{0.1}$. Or, nous observons, sur cette esquisse, que les décrochements $\mathrm{N}_{2} 0^{\circ}$ sont situés sur le flanc ouest du pli, où $\mathrm{S}_{0.1}$ est également $\mathrm{N}^{\circ}$ à $\mathrm{N} 20^{\circ}$, alors que les décrochements $\mathrm{N} 140^{\circ}$ affectent principalement le flanc est du pli dans leque] la foliation présente cette dernière direction.

Le mode d'évolution du panneau $\mathrm{D}$ permet de résumer l'histoire du glissement de Séchilienne de la manière suivante :

- action de la Romanche venue saper le versant au niveau de la " shear zone ") des " Ruines v, dans un secteur fragilisé par l'interférence de plusieurs familles de discontinuités. Un sillon important se forme dans le couloir de cisaillement:

- ouverture des fractures $\mathrm{N} 20^{\circ}$ et $\mathrm{N} 60^{\circ}$ à $\mathrm{N} 70^{\circ}$ en relation avec la décompression du massif (et avec la direction de contrainte actuelle pour les fractures subéquatoriales).

Les ouvertures observées sur les deux familles de discontinuités se faisant selon des directions perpendiculaires à celles-ci, les trajectoires des cibles dans le panneau D seront la résultante de ces directions. L'ouverture des fractures s'accompagne de l'affaissement en coin de la partie sommitale du versant (Fig. 15), affaissement autorisé par l'attitude des discontinuités subéquatoriales à pendage amont, ainsi qu'en attestent les mesures de nivellement effectuées dans la galerie de reconnaissance (cote 710 ) et qui rendent compte de la composante verticale des trajectoires des cibles. Dans le secteur mobile, où aucun plan de glissement n'a été repéré, les fractures $N 60^{\circ}$ à $N 70^{\circ}$, de même direction que les filons et à l'instar de ceux-ci, doivent s'ancrer profondément dans le massif, de sorte que la puissance de la zone déstabilisée pourrait être considé. rable.

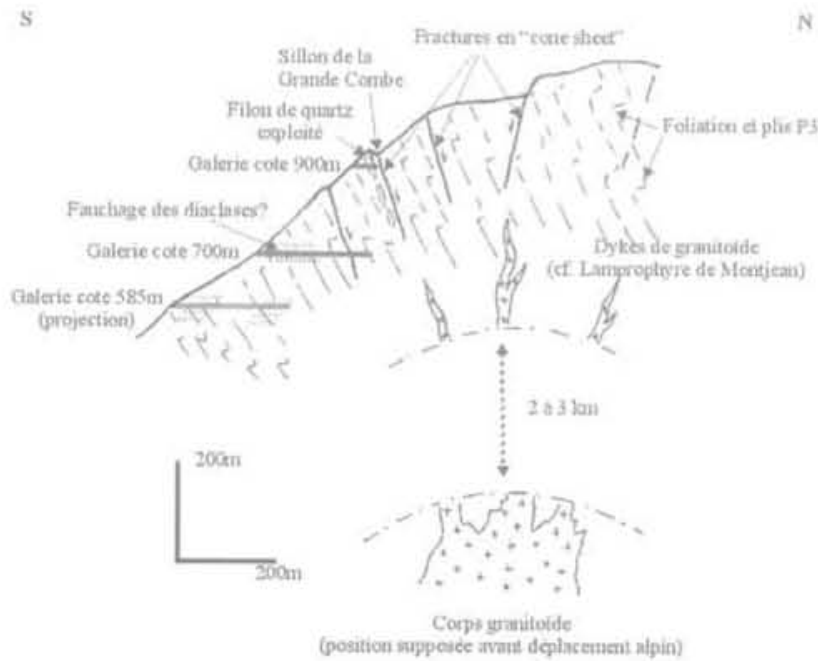

FG.15 Coupe interprétative N-S passant par le centre de l'affaissement du Mont Sec et montrant l'effet de coin.

Interpretation cross-section through the middle part of the "Mont Sec $n$ subsidence, showing the wedge action.

\section{Simulation numérique des mouvements de versant}

Compte tenu de l'état de fracturation du massif et du rôle apparemment joué par certaines familles de discontinuités la méthode retenue pour la simulation des déplacements est celle des éléments distincts (Cundall, 1976, 1991) dont on trouvera dans Vengeon (1998) une utilisation détaillée. Le type de modèle construit pour les simulations à l'aide du code de calcul UDEC est un modèle bidimensionnel, destiné à simuler l'effet de coin, qui s'appuie sur la connaissance structurale du massif développée précédemment. Il est construit sur une coupe du versant approximativement $\mathrm{N}-\mathrm{S}$, perpendiculaire au grand axe de l'ellipse constituée par l'affaissement sommital (Fig. 15).

\section{1}

\section{Le choix des discontinuités}

Les accidents utilisés dans le modèle sont respectivement :

- au niveau de l'affaissement sommital : la fracture arrière du Mont Sec (F) d'une quarantaine de mètres de hauteur, de direction $N 60^{\circ}$ à pendage $75^{\circ} \mathrm{S}$ et le sillon de la Grande Combe $\left(\mathrm{F}^{\prime}\right)$;

- dans le versant: deux fractures $N 60^{\circ}$ à N70 $\left.\mathrm{F}^{\prime \prime \prime}\right)$, à pendage $65^{\circ} \mathrm{N}$, bordées par un escarpement à regard nord, qui sont des répliques du sillon de la Grande Combe.

Ces quatre accidents, situés aux cotes respectives : $630,720,960$ et $1100 \mathrm{~m}$, découpent le versant en quatre compartiments C1, C2, C3 et C4 représentés par des grisés différents mais constitués de matériaux identiques (Fig. 16).

Les discontinuités conjuguées $\mathrm{N}^{\circ} 0^{\circ}$ et $\mathrm{N} 140^{\circ}$ ne sont pas prises en compte dans le modèle car elles sont subparallèles à la coupe considérée.

5

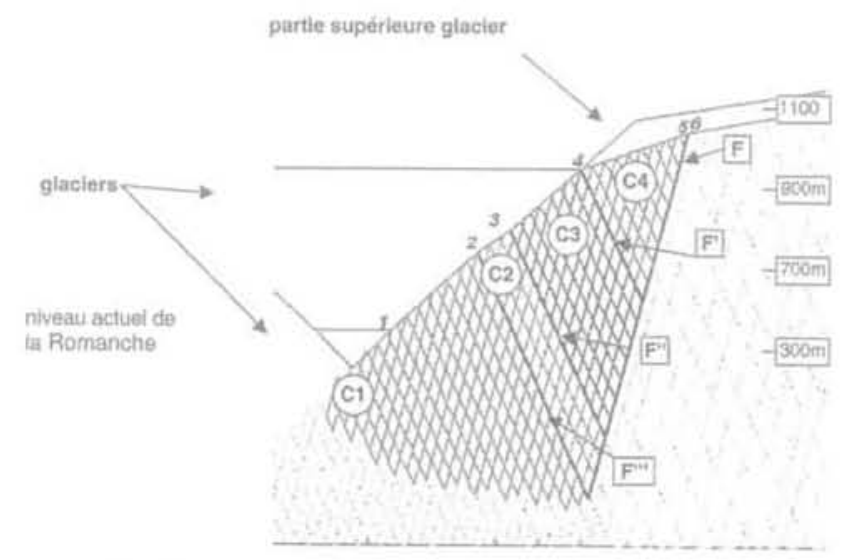

FG. 16 Modèle du versant de Séchilienne prenant en compte les différentes familles de discontinuités avec localisation des repères de déplacements $\left(\mathrm{n}^{\circ} 1\right.$ à 6$)$.

The slope Séchilienne model taking into account the different kinds of discontinuities, with location of bench marks ( $\mathrm{n}^{6} 1$ to 6 ). 


\section{Réalisation du modèle}

Le versant modélisé est situé entre les altitudes du bed-rock dans la vallée de la Romanche $(280 \mathrm{~m})$ et du Mont Sec (1 $100 \mathrm{~m})$. Le découpage du massif est complété, d'une part, par un réseau de fractures secondaires $\mathrm{N} 60^{\circ}$ à $\mathrm{N} 70^{\circ}$, à pendage $\mathrm{N} 65^{\circ}$, moins marquées dans le paysage mais observées sur le terrain, d'autre part par le réseau de diaclases de direction ${\mathrm{N} 100^{\circ}}^{\circ}$ $\mathrm{N} 120^{\circ}$ à pendage $75^{\circ} \mathrm{S}$ relevé en galerie. Le choix de ces deux familles de discontinuités permet de délimiter des panneaux rocheux en forme de losanges de $3000 \mathrm{~m}^{2}$ de superficie. Le nombre de blocs ainsi définis est de l'ordre de 600 pour l'ensemble du massif.

La continuité de la fracturation dans le massif à l'amont du modèle, permet de ne pas limiter les mécanismes mis en jeu dans les mouvements du versant au seul volume du glissement en les intégrant dans un environnement global. Les repères sélectionnés pour le suivi des déplacements verticaux et horizontaux pendant le calcul sont représentés par des numéros (Fig. 16).

\section{3}

\section{Propriétés des matériaux}

Les caractéristiques mécaniques affectées à la partie supérieure du versant, supposée la plus fracturée, sont différentes de celles de la base, considérée comme plus rigide. Les valeurs des modules d'Young et coefficients de Poisson sont donc respectivement :

$E_{\mathrm{F}}=5 \mathrm{GPa}$ et $v_{\mathrm{F}}=0,25$ en partie supérieure ;

$\mathrm{EB}=10 \mathrm{GPa}$ et $v_{\mathrm{B}}=0,35$ à la base.

Le poids volumique du massif est de $25 \mathrm{kN} / \mathrm{m}^{3}$, celui du glacier de $10 \mathrm{kN} / \mathrm{m}^{3}$. $20^{\circ}$.

L'angle de frottement entre les blocs vaut $\varphi_{\text {blocs }}=$

Les valeurs de l'angle de frottement de F, F', F' et $F^{\prime \prime \prime}$ sont égales à $\varphi_{\text {falles }}=10^{\circ}$. Ces valeurs sont choisies relativement faibles, d'une part en raison de l'altération argileuse observée dans les micaschistes, d'autre part pour être plus représentatives des caractéristiques résiduelles et tenir compte du caractère différé dans le temps des déplacements.

La cohésion et la résistance à la traction des joints sont respectivement prises égales à $j_{c o h}=10 \mathrm{kPa}$ et $j_{\text {rens }}=$ $1 \mathrm{kPa}$ et leurs raideurs normale et tangentielle égales à $1 \mathrm{GPa} / \mathrm{m}$. Ces valeurs $j_{\mathrm{coh}}$ et $j_{\text {tens }}$ sont prises volontairement faibles pour accentuer les déplacements entre les blocs et ainsi mieux visualiser les mécanismes.

Des séries de calculs paramétriques sont effectuées en faisant varier les valeurs des principaux paramètres: pendages, module d'Young et frottement des discontinuités principales.

\section{4}

\section{Conditions aux limites et chargement du modèle}

Après stabilisation du modèle sous l'effet de la pesanteur, les calculs sont réalisés pour trois configurations distinctes:
1) une configuration initiale: le glacier de la Romanche occupe la vallée jusqu'à l'altitude de $960 \mathrm{~m}$. Le sommet du versant du mont Sec est surmonté d'une calotte glaciaire d'une cinquantaine de mètres de hauteur;

2) après une première phase de déglaciation, une partie du glacier subsiste jusqu'à l'altitude $330 \mathrm{~m}$ (cote actuelle des alluvions) ;

3) après la deuxième phase de déglaciation la vallée est totalement vidée jusqu'au bed-rock $(280 \mathrm{~m})$.

\section{5}

\section{Résultats des simulations}

\section{$0.5 \mathrm{hl}$}

\section{Les déplacements verticaux}

Le glissement étant facilité par une valeur faible du frottement, on observe, dans le cas du modèle à fracturation réduite (au sud de F), un affaissement supérieur à $40 \mathrm{~m}$ en sommet de zone fracturée, comparable à la topographie observée actuellement (compartiment C4, Fig. 17). Ce modèle souligne le rôle prépondérant de la fracture F dans la direction et dans l'amplitude des déplacements des blocs de la partie aval du versant. L'arrangement des blocs, en partie supérieure du compartiment C4, détermine des marches d'escaliers, les glissements sur les failles $\mathrm{F}^{\prime}, \mathrm{F}^{\prime \prime}$ et $\mathrm{F}^{\prime \prime \prime}$ créant des escarpements à regard amont de quelques mètres de hauteur. L'affaissement en coin du compartiment supérieur C1 entraîne la déformation du versant et provoque son soulèvement d'une dizaine de mètres dans sa partie médiane.

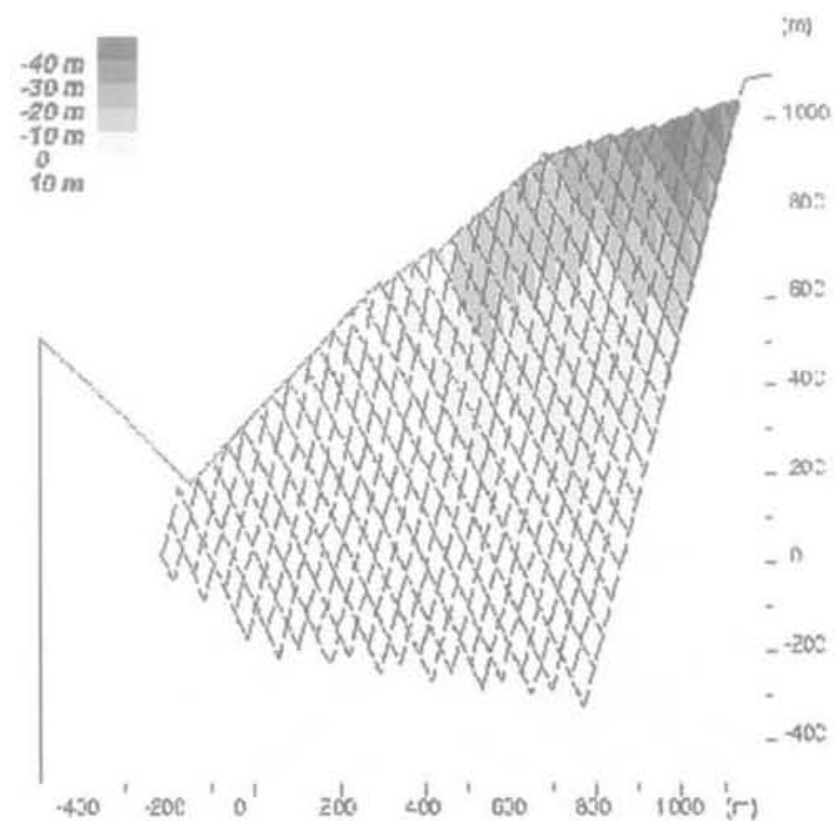

FG.17 Iso-déplacements verticaux dans le massif partiellement fracturé.

Vertical displacements in the partially fractured rock mass. 


\section{Les déplacements horizontaux}

Le glissement du compartiment sommital le long de la faille F impose des déplacements horizontaux vers la vallée. Pour un frottement faible, la simulation de ces derniers peut atteindre une amplitude supérieure à $40 \mathrm{~m}$ au niveau de la Grande Combe. Ils provoquent un tassement souligné par un escarpement entre les compartiments C3 et C4. Les mouvements horizontaux s'atténuent progressivement vers la base du versant (Fig. 18), engendrant une légère flexure des discontinuités à pendage nord.

\section{5 .3}

\section{La répartition des contraintes}

Les contraintes restent peu élevées dans la plus grande partie du versant. Elles sont logiquement les plus fortes à la pointe du cône renversé. En revanche

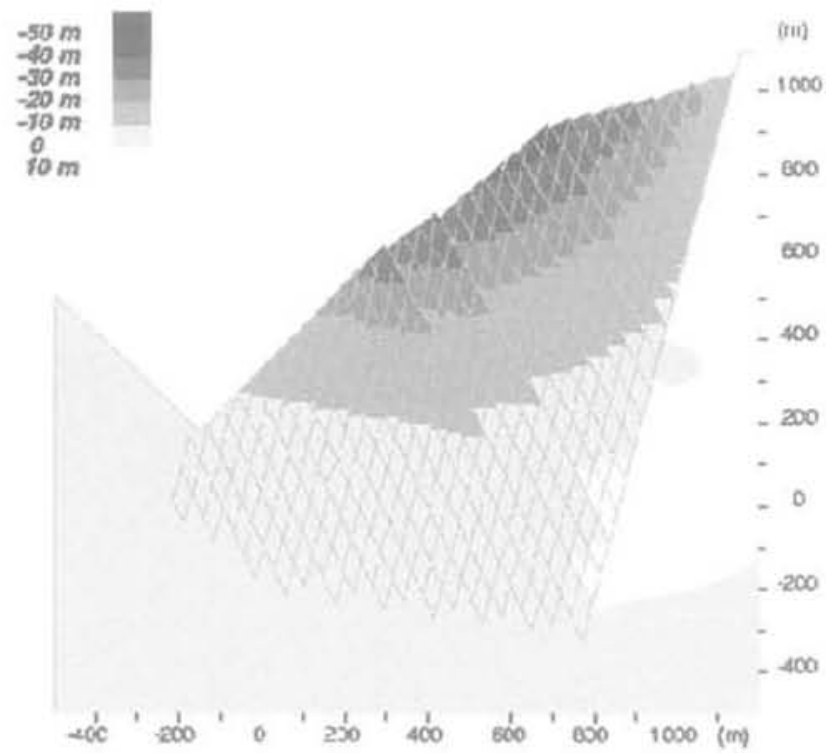

FIG. 18 Iso-déplacements horizontaux dans le massif partiellement fracturé.

Horizontal displacements in the partially fractured rock mass.

on note une décompression (légères tractions horizontales) à la base du versant, au voisinage de la surface (Fig. 19),

\section{5 .4}

\section{Étude paramétrique}

Sur le tableau I est figurée l'influence du frottement et de l'inclinaison des discontinuités sur la valeur des déplacements verticaux (cf. repères, 1, 2, 3, 4, 5 et 6 , figure 16).

\section{- Influence du frottement entre les blocs}

Pour une faible valeur du frottement $\left(\varphi=10^{\circ}\right)$ sur les failles $F, F^{\prime}, F^{\prime \prime}$ et $F^{\prime \prime \prime}$, les déplacements sont de l'ordre de grandeur des observations de terrain. En appliquant cette valeur à tout le versant, un affaissement généralisé se produit conduisant à un effondrement de $125 \mathrm{~m}$

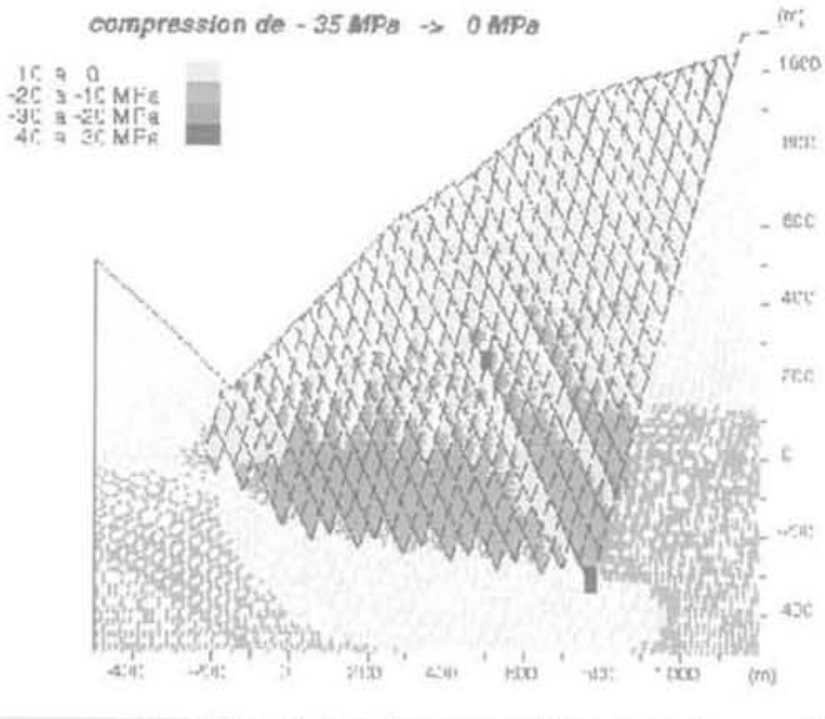

FG. 19 Répartition des contraintes dans le massif partiellement fracturé.

Stress distribution in the partially fractured rock mass.

au niveau du mont Sec. Pour une valeur $\varphi=20^{\circ}$ sur l'ensemble du modèle, les déplacements de blocs sont légèrement plus faibles que dans la réalité et les escarpements moins marqués.

\section{- Influence du pendage des discontinuités}

$\mathrm{Si}$ le pendage de la faille $\mathrm{F}$ passe de $75^{\circ}$ à $70^{\circ} \mathrm{S}$ et celui des failles $\mathrm{F}^{\prime}, \mathrm{F}^{\prime \prime}, \mathrm{F}^{\prime \prime \prime}$ de $65^{\circ}$ à $60^{\circ} \mathrm{N}$ les contraintes dans le modèle augmentent en partie inférieure des accidents principaux et les phénomènes de tractions en pied de versant disparaissent. L'affaissement sommital est également plus faible $(20 \mathrm{~m})$, tandis que le soulèvement du compartiment C1 est plus important.

\section{6}

\section{Comparaison avec les résultats du modèle Vengeon (1998)}

Tous deux réalisés à l'aide du code de calcul UDEC. les modèles Vengeon (1998) et LCPC ont en commun le

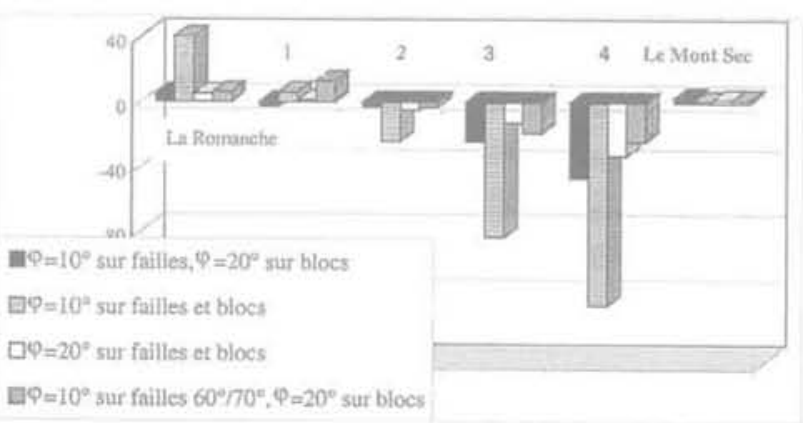

TABLEAU Déplacements verticaux des repères sur talus en fonction du frottement et du pendage des discontinuités.

Vertical displacements of bench marks as a fonction of friction angles and dip angles of discontinuities. 
mode de découpage du massif en blocs losangiques. Les principaux résultats du modèle Vengeon, basé sur un découpage par des fractures verticales ou à pendage aval (vers la vallée), sont les suivants :

- ouvertures répétitives de petits fossés d'effondrement:

- flexion de lanières avec ressaut à regard amont : - tassement progressif depuis la zone arrière stable du mont Sec jusqu'à la rupture de pente de la cote $900 \mathrm{~m}$.

Le modèle LCPC prend en compte, dans le découpage du massif, les fractures héritées de l'histoire tectonique de celui-ci, en particulier celles liées à la genèse du grand pli sommital et à la mise en place du champ filonien de Séchilienne : discontinuités en éventail (pendage amont et aval) et individualisation des failles majeures observées dans le versant. Les résultats diffèrent de ceux du modèle Vengeon par les points suivants :

- l'escarpement arrière du mont sec est plus marqué; - la subsidence du Mont Sec est assimilée à un affaissement en coin ;

- seuls les accidents majeurs sont matérialisés par un sillon bordé d'un escarpement à regard amont.

Ce modèle présente l'intérêt de reproduire relativement fidèlement la topographie actuelle du versant.

\section{7}

\section{Conclusion}

La complexité de l'histoire tectonique du versant de Séchilienne permet d'interpréter la « série satinée » comme une vaste bande de cisaillement (comprenant des zones de déformation ductile et des zones en extension), dans laquelle a pu se mettre en place le champ filonien de Vizille-Séchilienne dans des fractures à pendage centripète. Ce champ de fractures semble en relation avec la montée d'un corps granitoìde et la genèse d'un grand pli, par la suite découpé par un réseau de décrochements conjugués d'âge alpin.

Les structures et discontinuités principales impliquées dans le mouvement de versant sont respectivement :

- les filons de direction $N 60^{\circ}$ à $N 70^{\circ}$ et la fracturation associée ;

- le pli, sommital à grand rayon de courbure, d'axe $\mathrm{N} 20^{\circ}$, penté de $45^{\circ}$ vers le nord;

- les décrochements $\mathrm{N}-\mathrm{S}, \mathrm{N} 20^{\circ}$ et $\mathrm{N} 140^{\circ}$.

La combinaison de ces structures est à l'origine de deux types de désordres:

- l'écroulement actif du couloir des « Ruines »;
- l'affaissement sommital du Mont Sec.

La décompression du versant, consécutive au retrait du glacier de la Romanche, a favorisé l'ouverture de ces différentes familles de discontinuités, la mise en mouvement de panneaux à cinématique variable et l'effondrement en coin de la partie sommitale.

La présence supposée d'indices de néotectonique sur la vallée de la Romanche peut permettre d'envisager un rôle éventuel de l'activité sismique dans le déclenchement ou l'accentuation des instabilités observées.

Les simulations numériques ont été réalisées sur un modèle 2D d'une section perpendiculaire aux discontinuités de directions subéquatoriales, construit sur les éléments structuraux régionaux, en particulier sur les structures les plus fortement impliquées dans la genèse des désordres.

La prise en compte des fractures principales (F, F', $\left.F^{\prime \prime}, F^{\prime \prime \prime}\right)$ associées à des valeurs de paramètres physiques permet de simuler une évolution du versant comparable aux observations morphologiques, autant dans l'existence d'une falaise d'une hauteur notable, délimitant le plateau nord, que dans la présence d'escarpements à regard amont au droit des failles majeures à pendage nord.

Des calculs paramétriques ont montré que les diffèrences d'amplitudes des déformations dépendent essentiellement des caractéristiques mécaniques des plans de discontinuités et de leur pendage. Cependant, les mécanismes, de même que le comportement global du versant restent sensiblement les mêmes dans les différentes simulations : affaissement en coin de la zone supérieure et soulèvement de la partie inférieure, dénivelées en escaliers plus ou moins marquées, fléchissement (ou fauchage) des ( bancs $)$ délimités par chaque famille de discontinuités.

D'ambition limitée, du fait notamment de sa nature bidimensionnelle, le modèle du versant instable du Mont Sec utilisé dans cette étude, permet néanmoins de distinguer les discontinuités principales influençant la déformation du massif et de souligner l'intérêt que revêt la reconnaissance géologique approfondie de tout massif en préalable à l'étape de modélisation.

Le risque à court terme consiste en la poursuite de l'évolution de la partie haute du couloir des « Ruines $n$ par détachement de masses de quelques dizaines, voire centaines de milliers de mètres cubes. Le départ brutal de la zone considérée comme très active, située en partie supérieure NW du couloir des « Ruines » constitue également une menace à court terme. Le volume de cette zone (d'une superficie de 4 à $5.10^{4} \mathrm{~m}^{2}$ ) est de l'ordre de $2,5.10^{6} \mathrm{~m}^{3}$. Une inconnue subsiste sur le comportement du reste du versant instable en cas de départ de cette masse. 
Adam D., Gagny C. - " Contrôle structural et magmatique de la formation de gisements d'étain-tungstène du Tras os Montes oriental (Portugal). Guides pour l'exploration minière s. Chronique de la Recherche Minière n 496, 1989, p. 57-74, 17 fig.

Alfonsi P., Durville J.L. Rachez X. "Quelques applications de la méthode des éléments distincts en mécanique des roches ». Bulletin de Liaison des Laboratoires des Ponts et Chaussées $n^{\circ} 214.1998$, p. 31-43.

Antoine P., Camporota P., Giraud A., Rochet $L_{\text {. }}-a$ La menace d'écroulement des "Ruines" de Séchilienne (Isère) y. Bulletin de Liaison des Laboratoires des Ponts et Chaussées n ${ }^{\circ}$ 150-151, 1987. p. 55-64.
Cundall P. - - Computer interactive graphics and the Distinct Element Method. in Rock Engineering for fondations and slope ». Proc. ASCE Sp. Conf., Boulder (Colorado), vol. 2, 1976, p. 193.199 Cundall P.. Hart R.D. - « Numerical modelling of continua n. Engineering Computations vol. 9, 2, 1991, p. 101-113.

Effendiantz L., Guillot S. - "Galerie hyciraulique et de reconnaissance géologique de Séchilienne. Rapport géologique n. CETE de Lyon, LRPC RhôneAlpes, Université Lyon 1-CNRS, Laboratoire de dynamique de la lithosphère, 2000.

Evrard H., Gouin T. Benoit A.. Duranthon J.P. - « Séchilienne. Risques majeurs d'éboulements en masse. Point sur la surveiflance du site m. Bulletin de Liaison des Laboratoires des Ponts et Chaussées, $n^{\circ} 65,1990$, p. $7-16$

Guillot S. - "Les Massifs cristallins externes alpins revisités 3. Proc. RST 2000, 17 au 20 avril, Cité des Sciences et de l'Industrie, La Villette, Paris, 2000.

Potherat P., Guillot S., Effendiantz L.. Delacourt $\mathrm{C}$. - « Champ de déformation actuel et rồle de la néotectonique dans les mouvements de versant de Séchilienne \%. En préparation, 2001.

Thourvenot, F. Fréchet J. Jennatton L «The Beljedonne fault: Identification of a major active seismic strike-slip fault in the western Alps s. En préparation, 2001

Vengeon J.M. - " Déformation et rupture des versants en terrain métamorphique anisotrope $x$. Thése de l'Unfversité Joseph-Fourier, Grenoble 1, 1998. 